\title{
AC 2008-575: CHILDREN'S EXPERIENCE WITH CONSTRUCTION SETS: EARLY WARNINGS OF ENGINEERING INTEREST AMONG GIRLS?
}

\section{Cortney Martin, Virginia Polytechnic Institute and State University}

Dr. Cortney Martin has worked in information design, usability, and education for over 15 years including serving as the Assistant Director of the Blacksburg Electronic Village and the Broadband Wireless Networking Director for Virginia Tech. She teaches as a part of an innovative interdisciplinary thematic four-course sequence focused on Earth Sustainability. Her $\mathrm{PhD}$ is in Industrial Engineering (human factors) from Virginia Tech.

\section{Tonya Smith-Jackson, Virginia Polytechnic Institute and State University}

Dr. Tonya L. Smith-Jackson is an Associate Professor and Director of the Assessment and Cognitive Ergonomics Lab in the Grado Department of Industrial and Systems Engineering and at Virginia Tech. She received her Ph.D. from North Carolina State University in 1998. 


\title{
Children's Experience with Construction Sets: Early Warnings of Engineering Interest Among Girls?
}

\begin{abstract}
This mixed-method study explored gender differences among children ages six and nine years as they worked with common interlocking building toys and their accompanying assembly instructions. There were no differences in the speed with which girls and boys assembled the toys; however the six-year-old girls were less accurate than the six-year-old boys. This difference disappeared by age nine resulting in similar performance levels. However, by age nine, girls reported having less fun than boys and they perceived assembly as more difficult. Girls tended to blame difficulties on themselves while boys were more likely to blame the instructions. The results highlight the importance of carefully selecting appropriate building and construction sets with well-designed instructions, particularly for girls, to encourage and not discourage them from participating in these important building and manipulative activities. It further suggests that STEM instruction methods for young girls should provide opportunities for girls to bolster their confidence with hands-on construction activities.
\end{abstract}

\section{Introduction}

One early exposure children have to engineering principals and design is through interactions with construction models and building toys such as interlocking bricks, logs, and figures. The colorful, tactile objects awaken the senses and the mind, and promote motor, cognitive, emotional, and social development in children ${ }^{1,2}$. Among all toy categories, building sets had the largest rate of sales increase at $16 \%$, growing from $\$ 599.8 \mathrm{M}$ in 2004 to $\$ 695.2 \mathrm{M}$ in $2005^{3}$.

Building kits also expose children to the language of graphical assembly instruction, which is becoming more ubiquitous largely because of globalization of product markets. Unfortunately, when instructions are difficult to follow, adults often think the fault lies with them and that they are not skilled or experienced enough for the task ${ }^{4}$. How might this self-blame impact children, particularly girls, who in academic domains are more likely to attribute failure to lack of ability ${ }^{5}$ than boys.

There is some empirical evidence that experiences with building toys can lead to enhanced spatial abilities that may influence aptitudes in math and science ${ }^{6,7,8}$. Based on the potential linkage between toy assembly and STEM areas, it is important to understand how boys and girls interact with instruction-based assembly to be sure both populations are being well-served.

There are few studies to draw from regarding children and object assembly and the literature presents conflicting evidence about the effects of gender. Murphy and Wood ${ }^{9}$ in their study of four through eight-year-old children found gender differences to be far greater even than agerelated differences between the four through five-year-olds and eight year olds. The results showed that girls paid more attention to the photo instructions, were far more efficient with operations, and performed the pyramid building task more quickly than the boys. The boys tended to use a strategy based on trial and error, with less reliance on the photos. The boys' trial 
and error strategies caused them to complete the task either very quickly, or very slowly, while girls were more consistently reliable. Stufft ${ }^{10}$ found gender differences interacted with age. Males in grade four outperformed their female counterparts, but this difference disappeared in grade six, and reversed in grade eight, with females performing better.

Women beginning engineering programs typically exhibit relatively high-levels of selfefficacy ${ }^{11}$. To address stagnant enrollments among women, it is important to understand and mitigate events that may undermine development of self-efficacy in girls. Could hands-on assembly be an activity that can encourage or discourage girls from the paths to STEM careers? To begin to understand the connection, this research sought to identify if gender-related differences existed in toy assembly performance, subjective evaluations, and indications of selfefficacy.

\section{Methods}

\section{Participants}

Twenty-four children participated in the study including six each of six-year-old girls $(M=5.92$, $S D=0.30)$, six-year-old boys $(M=5.90, S D=0.20)$, nine-year-old girls $(M=9.01, S D=0.21)$, and nine-year-old boys $(M=8.98, S D=0.32)$. Participants were drawn from the community surrounding the university and from local after-school programs via flyers and posters. Parents certified that their children did not have visual, auditory, or motor difficulties, or learning disabilities that could impact performance.

\section{Toys and Instructions}

Four toys and their unmodified, manufacturer-provided pictorial assembly instructions were used in the study: the Kid K'NEX® Fish-Eyed Friends; Lincoln Logs® Frontier Junction; the LEGO® X-Pod (\#4349) dragonfly design; and BIONICLE® Rahaga Bomonga (\#4878). These toys and instruction sets were selected because they represented a variety of designs ranging from the BIONICLE's single-sheet, multi-frame, grayscale design to the LEGO's booklet style, and the K'NEX flat assembly with redundant shape and color coding (see Table 1). For reasons outside the scope of this paper, two different models from the K'NEX set were included in the study for a total of five construction tasks. All toys were age-appropriate according to the manufacturer's age labeling with the exception of the BIONICLE that was labeled for children seven and older. 
Table 1. Objects and Instructions Used for Assembly Tasks

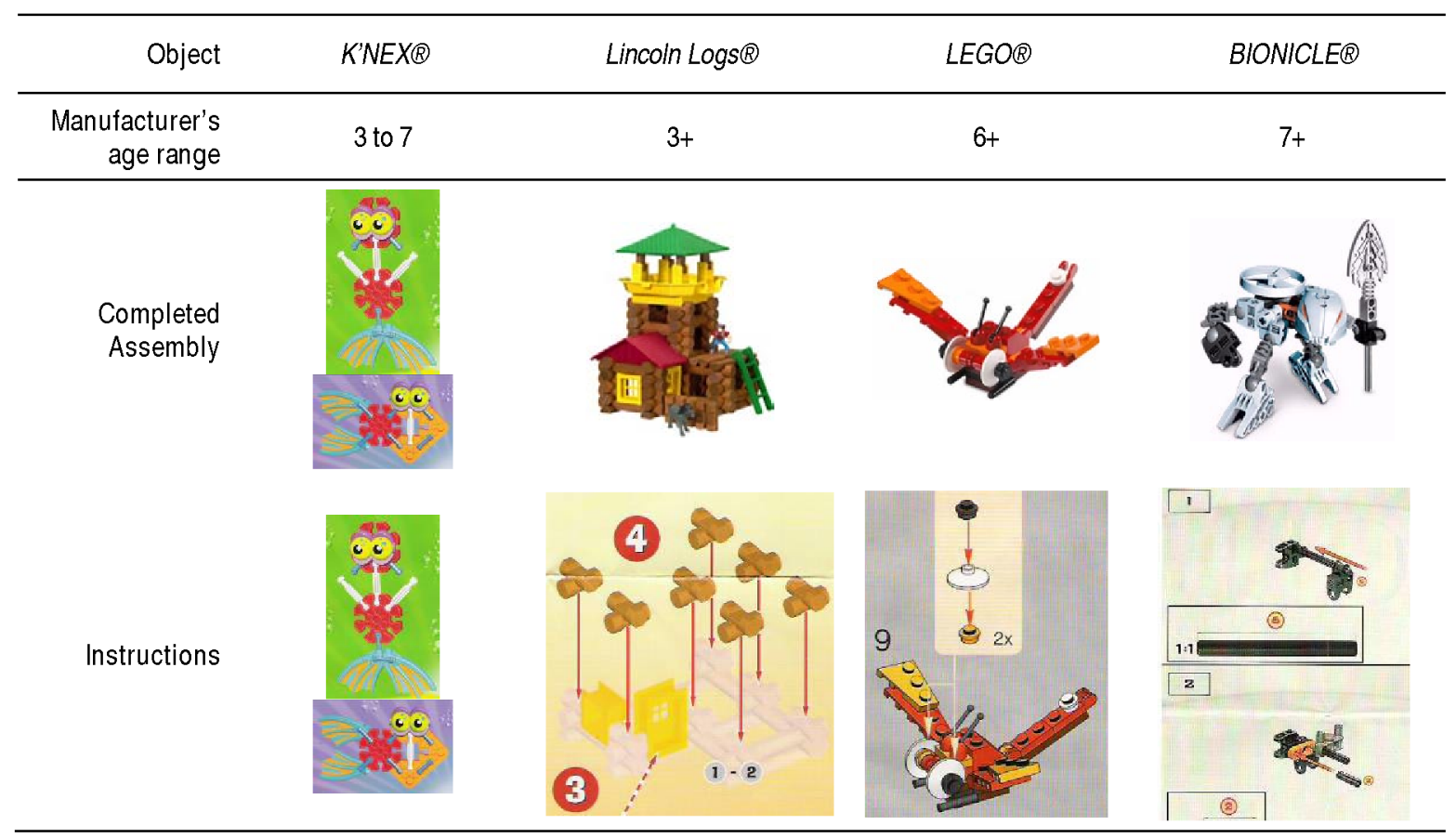

\section{Procedure}

At the start of the session, prior building experience was assessed by asking children to indicate whether they had played with eight types of building toys a lot, a little, or not at all and these responses were totaled for a numeric measure of previous experience ranging from 0 to 24 . Then each child was allowed up to twelve minutes to work with each of the five assemblies. Several of the assemblies could be completed in just a few minutes so the entire session was completed in under an hour. The presentation order was partially counterbalanced to offset practice effects. After each assembly, children were asked to rate the fun, frustration level, and ease of the task using a visual analog scale anchored with faces, as shown in Figure 1.

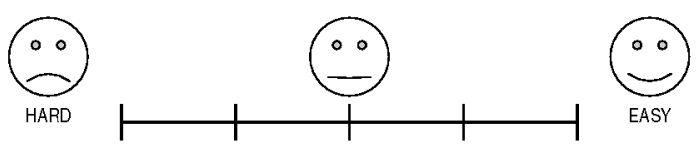

Figure 1. Example of the rating scale.

Children were instructed to think-aloud ${ }^{12,13}$ while assembling to better assess mental models and processes. Sessions were videotaped and later evaluated to measure assembly time, track errors, and to transcribe verbal protocols. At the conclusion of the session, participants selected a toy prize and were given a Polaroid photo of their assemblies. 


\section{Results}

Because of the relatively unexplored nature of the problem, both quantitative and qualitative analyses were used to obtain a more complete understanding of how boys and girls work with assembly instructions and tasks. Highlights of gender-related findings are reported here, but more complete results are available ${ }^{14}$.

\section{Previous experience}

A two-way factorial ANOVA on age and gender revealed a significant difference in previous building experience due to gender $(F[1,20]=13.47, p=.002)$ but not age. Boys $(M=7.42, S D=$ 2.57) had more experience than girls $(M=4.08, S D=1.93)$. Despite differences in experience, boys and girls produced assemblies at similar speeds. However, there were notable differences in task accuracy and task ratings.

\section{Error rates}

Error rates were evaluated using the nonparametric Kruskal-Wallis one-way analysis of variance test due because just over half of the assemblies had zero errors resulting in a non-normal distribution. Analyses showed significant differences in the number of incorrect parts by age $\left(\chi^{2}\right.$ $[4, N=120]=16.02, p<0.0001)$ and gender $\left(\chi^{2}[1, N=120]=4.47, p=0.0345\right)$. Comparisons among age groups by gender and among gender groups by age revealed differences (with Bonferroni adjusted $\alpha=0.025)$ between the six-year-old and nine-year-old girls $\left(\chi^{2}[1, N=60]=\right.$ $12.38, p=0.0004)$ as well as between the six-year-old girls and six-year-old boys $\left(\chi^{2}[1, N=60]=\right.$ 5.42, $p=0.0199$ ). This represented an Age $\mathrm{x}$ Gender interaction in which the six-year-old girls had higher median errors than the other groups (Figure 2). Previous experience also impacted error rates, $\left(\chi^{2}[1, N=120]=3.50, p=0.061\right)$, but was less significant than gender.

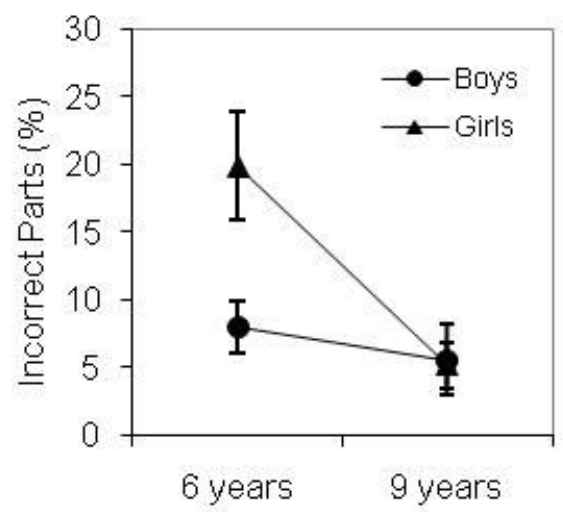

Figure 2. Mean $(+\mathrm{SE})$ percent of incorrect parts by age and gender $(n=30)$.

\section{Ratings of Fun}

An Age x Gender interaction was found for ratings of fun, $F(1,20)=4.61, p=0.04$, as depicted in Figure 3. There were significant differences between the nine-year-old boys and girls ( $F[1$, 
$20]=4.24, p=0.52)$ as well as between the six-year-old and nine-year-old girls $(F[1,20]=$ $3.34, p=0.08)$. In other words, gender differences were present in the nine-year-olds but not the six-year-olds and age differences existed for girls but not for boys. Previous experience had no effect on ratings-fun.



Figure 3. Mean ratings of fun $(+\mathrm{SE})$ showing interaction effect of Age $x$ Gender with differences between the age groups of girls as well as between the nine-year-olds $(n=30)$

\section{Ratings of ease}

A main effect of gender showed that boys rated the toys as easier $(M=7.13, S D=3.60)$ than girls $(M=5.55, S D=4.00)$ did. Extent of previous experience had no effect on ratings-ease.

\section{Qualitative analysis}

The qualitative analysis employed Grounded Theory ${ }^{15,16}$ methods. This is an inductive process for extracting relationships and models and is suited to areas of inquiry that are not well researched. The data was coded using a commercial software package for qualitative content analysis, Atlas.ti (www.atlasti.com).

Children expressed varying levels of self-efficacy, the self-perception of one's ability to complete a given task. Although self-efficacy was not measured directly, based on Bandura' ${ }^{17}$ social cognitive theories, positive, affirming, motivated, and confident statements were interpreted as indicative of high self-efficacy and instances of negative, pessimistic, disengaged, anxiety-related statements were associated with low self-efficacy.

The most significant gender-related findings were: (1) of equally skilled girls and boys, girls were more likely to attribute difficulty to themselves rather than the toy; (2) difficult masculineoriented building toys negatively impacted self-efficacy to a greater extent among girls; and (3) girls tended to be more process-oriented while boys were more outcomes-oriented.

\section{Self-efficacy and attributions}

Statements of high self-efficacy were uttered more often by boys than girls. For instance, a nineyear old boy describes himself as he works with the LEGOs, "I'm always good at building things even if I get a hard time.... just whiz through it like it's nothing...I'm always good at building 
things even if I get a hard time." And a six-year-old boy described himself as, "the little train that could."

Low self-efficacy statements were far more common than statements of high self-efficacy and the most striking examples of low self-efficacy came from the nine-year-old girls. Each of the following examples, all uttered by nine-year-old girls, also demonstrates how they use internal attributions, where failure is directed inward, attributed to one's self.

- "Did I mess up? I built one of these before and I really messed up."

- "I'm going to fail this one...I'm telling you, I can't do BIONICLEs."

- "I might make it, I said might, which means I probably won't."

- “Oh, I'm not good with doors, I don't like doors. I'm just not good with them. I never put them in the right place...I did something wrong....Argh! I'm bad with these!"

In contrast, other statements indicated an outwardly-focused blame or external attribution for difficulty directed toward the toy or instructions. There were ten examples of; eight were from the boys and just two from six-year-old girls. The following were uttered by boys:

- "Now this is harder because you actually have to find the pieces. And it's hard to find the pieces, which don't like to be found. They seriously don't, or is this just missing a piece...oh there it is."

- "I think I might not have to look at the directions because I think the directions are wrong. I do not know what is wrong with these directions. I'm just trying whatever I think cause it does not make sense. I think the directions are wrong, from what I see now."

- "It never said to put this on. Well... yeah, it never said."

- "I think the directions are wrong from what I see now, nope, they're wrong."

\section{Masculine toys}

The BIONICLE represented a much more masculine toy than the others and elicited some strong responses from a number of girls. One nine-year-old girl, who had little difficulty building the K'NEX animals correctly, reacted to this toy by responding "Oh, I'm not good with BIONICLEs." When asked if she had done one before, she replied, "No." Perhaps because it did not appeal to her, she also felt she could not do it. Subsequent comments seemed to support her initial impression.

I don't even know the instructions...They're really hard to put together. I'm never going to get this done, I can't even get the first step right....I'm not good at BIONICLEs. I don't know how boys do this...I think I might have gotten it, almost...all the boys from my school could do this...I did it wrong...I don't like using instructions yet...gosh this is really hard...oh gosh they all look harder!

The previous example also demonstrated a gender-based social comparison. In this study, all instances of gender comparisons were of older girls referencing against boys. This occurred most often with regard to the BIONICLE, which was alluded to as being for boys. "I don't like 
them," said one nine-year-old girl, "Not many girls do." Another participant elaborated and suggested that boys were endowed with some special skill that enabled them to assemble the toy:

I'm not good with BIONICLEs, I don't know how boys do this. Did the last boy you had do this?' 'Cause most boys can do this. I know a lot of boys at my school do it, It's like amazing. Did the last boy you had do this? 'Cause most boys can do this.

Some of the boys indicated that they learned about BIONICLE assembly through a relative or classmate. Because BIONICLEs are desired by boys, they are motivated to learn about them and it is much more a part of the boys' culture than the girls' culture. Girls just see that boys are successful but do not take into account the boys' prior experience. In this way, girls may believe that boys have a special talent for reading instructions and assembling toys. The importance of social comparison is that designers cannot rely on peer groups to compensate for poor instructions by educating one another. This disenfranchises individuals outside the group for whom the instructions are the only means for successful assembly.

\section{Focus on process}

Differences in assembly goals were evident by error recovery strategies. Some children were more concerned with having correct assembly rather than following the procedure faithfully. Upon recognizing an error, they tended to deconstruct minimally and were willing to diverge from the instructions to make the correction. For others, the priority was to adhere to the assembly process accurately. When they discovered an error, this group of children tended to deconstruct far more than necessary to stay true to the process. In some cases, a single error would drive the children to restart the entire assembly from the first step. Five girls and two boys showed evidence of focusing on the process rather than the outcome.

\section{Discussion}

To summarize, this study showed girls to be on-par with boys in terms of assembly speed, and while they may be lagging behind on accuracy at age six, they do catch up and match accuracy levels of boys by age nine. By age nine, girls indicate that they are having less fun with construction toys and they perceive assembly as more difficult. In addition, when girls have difficulty, they tend to blame themselves where boys are more likely to blame the instructions. The gender split is amplified when the toy in question is masculine. Boys have informal help networks that enable them to accomplish the assembly task. Girls, in the absence of this peer network of task experts, may be getting the message that boys have an innate advantage in construction tasks.

It is important that instruction developers create well-designed and thoroughly tested instructions, particularly for girls who tend to blame themselves when assembly is difficult. The observed gender difference in attributions aligns with findings regarding children's perceptions of mathematical successes and failures. Stipek and Gralinski ${ }^{5}$ found that among third-graders (typically around 8 years old), girls attributed failure to low ability more often than did boys. In addition, girls were more likely to have feelings of shame when encountering difficulties ${ }^{18}$.

Thus, poorly-designed or overly-difficult instructions may negatively affect girls to a larger extent than boys causing girls to avoid construction tasks. 
The tendency for girls to adhere more closely to instructions is also supported by other research. Studies of mathematical problem-solving strategies among revealed that first-grade girls were more likely to solve problems with concrete, overt methods like counting or using fingers; boys were more apt to work from memory ${ }^{19}$. First through third-grade boys showed a greater conceptual understanding of problem solving and were better able to solve novel problems whereas girls employed learned procedures and had more difficulty modifying the procedures to solve novel problems ${ }^{20}$. Boys may be more willing and able to compensate for confusing or poorly-designed instructions.

Girls need access to girl-oriented building toys or, at minimum, androgynous toys to reduce the negative gender comparisons and to foster feelings of greater self-efficacy. When a child believes a toy is intended for the opposite gender, they generally like it less ${ }^{21}$ which can impact the child's motivation and drive to overcome difficulty.

In addition to providing well designed, teachers and parents are encouraged to select toys and instructions that are at an appropriate level of difficulty. Age labels do not provide sufficient guidance. In fact, it may be appropriate to consider adding skill level labeling to construction kits, much in the way model kits have done for years. This would allow for individual goalsetting and staged learning.

It is also important that children have construction opportunities made available to them and that they have help and encouragement available. Girls need to be drawn into hands-on building activities with enticing toys that appeal to them. And perhaps it is time to offer girls-only LEGO leagues and similar activities. Poorly designed instructions, lack of opportunity, and a lack of support may combine to unwittingly drive girls away from construction sets and instructionbased assembly, and further down the line, STEM-based education and careers. This is an area worth further exploration and action.

\section{Limitations}

The child participants in this study were drawn from the relatively homogeneous population of a university town and are not necessarily representative of other groups, ethnicities, or cultures. In addition, the children who volunteered may be more likely to enjoy building tasks or have more experience with assembly than the larger population of children their age. Extensions of this study should draw upon a more diverse group of children.

\section{Acknowledgements}

This work was partially supported by Advance VT through the Virginia Tech Graduate School and the National Science Foundation ADVANCE Grant No. SBE-0244916. 


\section{References}

1. Brouwer-Janse, M. D., Suri, J. F., Yawitz, M., deVries, G., Fozard, J. L., \& Coleman, R. (1997). User interfaces for young and old. Interactions, 4(2), 34-46.

2. Eisenberg, M. E., Ann Nishioka. (1999). Middle tech: blurring the division between high and low tech in education. In A. Druin (Ed.), The Design of Children's Technology (pp. 243-273). San Francisco, CA: Morgan Kaufmann Publishers, Inc.

3. Toy Industry Association Inc. (2005). A year in review: the growth, challenges, and opportunities of the toy industry. New York, NY: Toy Industry Association, Inc.o. Document Number)

4. Adams, A. (1999). Usability testing in information design. In H. J. G. Zwaga, T. Boersema \& H. C. M. Hoonhout (Eds.), Visual information for everyday use (pp. 3-20). London: Taylor \& Francis.

5. Stipek, D. J., \& Gralinski, J. H. (1991). Gender differences in children's achievment-related beliefs and emotional responses to success and failure in mathematics. Journal of Educational Psychology, 83(3), $361-371$.

6. Robert, M., \& Heroux, G. (2004). Visuo-spatial play experience: Forerunner of visuo-spatial achievement in preadolescent and adolescent boys and girls? Infant and Child Development, 13(1), 49-78.

7. Tracy, D. M. (1987). Toys, spatial ability, and science and mathematics achievement: Are they related? Sex Roles, 17(3 - 4), 115-138.

8. Tracy, D. M. (1990). Toy-playing behavior, sex-role orientation, spatial ability, and science achievement. Journal of Research in Science Teaching, 27, 637-649.

9. Murphy, C. M., \& Wood, D. J. (1981). Learning from pictures: the use of pictorial information by young children. Journal of Experimental Child Psychology, 32, 279-297.

10. Stufft, D. L. (1988). The Effects Of Cognitive Style And Pictorial Procedural Instructions On Speed And Accuracy Of Assembly Performance By Middle School-Aged Children. Unpublished dissertation. Indiana University of Pennsylvania.

11. O'Hare, S. (1995). Freshman women in engineering: Comparison of their backgrounds, abilities, values, and goals with science and humanities majors. Journal of Women and Minorities in Science and Engineering, 2, $33-$ 47.

12. Ericsson, K. A., \& Simon, H. A. (1993). Protocol analysis: verbal reports as data. Cambridge, Mass: MIT Press.

13. Smith-Jackson, T. L. (2002, June 10-13). Child-Centered Safety Research Issues. Paper presented at the Proceedings of the XVI Annual International Occupational Ergonomics and Safety Conference, Toronto, Ontario, Canada, June 10-13.

14. Martin, C. V. (2007). Usability of pictorial assembly instructions for young children. University Libraries, Virginia Polytechnic Institute and State University, Blacksburg, Va.

15. Strauss, A., \& Corbin, J. (1998). Basics of qualitative research: Techniques and procedures for developing grounded theory. Thousand Oaks, California: SAGE Publications, Inc.

16. Glaser, B., \& Strauss, A. (1967). The discovery of grounded theory: Strategies for qualitative research. Chicago: Aldine.

17. Bandura, A. (1986). Social foundations of thought and action: a social cognitive theory. Englewood Cliffs, NJ: Prentice Hall.

18. Weiner, B. (1986). An attributional theory of motivation and emotion. New York: Springer-Verlag.

19. Carr, M., \& Jessup, D. L. (1997). Gender differences in first-grade mathematics strategy use: social and metacognitive influences. Journal of Educational Psychology, 89, 318-328.

20. Fennema, E., Carpenter, T. P., Jacobs, V. R., Franke, M. L., \& Levi, L. W. (1998). A longitudinal study of gender differences in young children's mathematical thinking. Educational researcher, 27(5), 6-11.

21. Martin, C. L., Eisenbud, L., \& Rose, H. (1995). Children's gender-based reasoning about toys. Child Development, 66, 1453-1471. 